\title{
Riesgo de contaminación por Cronobacter Sakazakii en leches en polvo para la nutrición de lactantes
}

\author{
Risk of Cronobacter Sakazakii \\ contamination in powdered \\ milk for infant nutrition
}

\begin{abstract}
Introduction: Cronobacter spp. is a bacterial genus that includes 7 species; Cronobacter sakazakii is the clinical specie that is the most reported and associated with meningitis and septicemia in infants. Given that it is transmitted by powdered infant formula (PIF), the WHO recommends that this product be free of Cronobacter, whereas the Chilean Food Sanitary Regulation (RSA) does not consider it. Objective: To assess the risk of C. sakazakii in PIF for consumption by infants. Methodology: A total of 72 PIF samples were analyzed using three brands originating from three countries. Aerobic plate count (APC), Enterobacteriaceae (ENT), and most probable number $(\mathrm{MPN})$ were performed using the methodology described by Puch and Ito (2001). Cronobacter differential agar was used to isolate strains (DFI, Oxoid, England), and the ID32E biochemical kit (Biomeriux, France) was used for phenotyping. The pathogen was identified and genotyped by multilocus sequence typing (MLST) based on the criteria found at http://www.pubm/st. org/cronobacter. Results: Median APC for step 1 and preterm PIF was $300 \mathrm{CFU} / \mathrm{g}$ (10-36 000) and $650 \mathrm{CFU}$ (70-30 000), respectively, and was higher in Chilean PIF $(p=0.016)$. There were no significant differences for type, country, or PIF brand in $75 \mathrm{CFU} / \mathrm{g}$ (10-36 000) and 200 CFU/g (10-1 000) ETN (p>0.05). Two strains from two different lots with characteristic strains in DFI agar were identified as C. sakazakii with 0.23 and $2.3 \mathrm{MPN} / \mathrm{g}$. In addition, Franconibacter helveticus, specie closely related to Cronobacter spp, was found in two other strains. Conclusions: The prevalence of Cronobacter sakazakii in all the samples was $2.7 \%$ isolated only in PIFs manufactured in Chile. The absence of Cronobacter spp in $25 \mathrm{~g}$ must be included in the Chilean RSA.
\end{abstract}

Key words: Cronobacter sakazakii, powdered infant formula, infants.

\section{INTRODUCCIÓN}

En Chile, en el comercio existen actualmente 2 tipos de fórmulas infantiles para la nutrición infantil: líquidas listas para el uso, con un buen nivel de inocuidad, pero de menor consumo; y en polvo (LP), que son las más utilizadas a nivel mundial pero no estériles (1). Garantizar su inocuidad microbiológica es de gran preocupación para los organismos reguladores y productores, ya que están destinados a recién nacidos y niños que tienen un sistema inmunológico aún poco desarrollado y una microbiota intestinal poco
Julio Parra F. $(1,2)$

Leslie Oliveras V. (2)

Alejandra Rodriguez F. $(1,2)$

Francisca Riffo S. (3)

Emily Jackson (4)

Stephen Forsythe (4)

(1) Departamento de Nutrición y Salud Pública, Universidad del Bío-Bío, Chillán, Chile (2) Magister en Salud Pública, Universidad del Bío-Bío, Chillán, Chile. (3) Escuela de Medicina, Universidad de Concepción, Concepción, Chile. (4) Pathogen Research Centre, School of Science and Technology, Nottingham Trent University, Clifton Lane, Nottingham, UK.

Dirigir la correspondencia a:

Julio Parra F Nutricionista Magister en Salud Públic
Departamento Nutrición y Salud Públic Universidad del Bío-Bío, sede Chillán Chillán, Chile CP 3780000

Tel: (56)42-2463107 - Fax 42-2463132 Email: juparra@ubiobio.cl

Este trabajo fue recibido el 9 de Diciembre de 2014 y aceptado para ser publicado el 25 de Enero de 2015.

competitiva (2).

Cronobacter spp fue inicialmente definido como Enterobacter sakazakii por Farmer y cols (3) como un nuevo género bacteriano, y clasificado posteriormente por Iversen y cols como Cronobacter spp (4). Joseph y cols en 2012 lo reclasificaron como Cronobacter spp con 7 especies que son: C. sakazakii, C. malonaticus, C. universalis, C. turicensis, C. muytjensii, C. dublinensis, C. condimenti (5).

Cronobacter sakazakii es considerado la especie más agresiva en individuos hipersensibles, siendo los grupos de 
población más afectados recién nacidos y adultos mayores, pero con mayor incidencia y gravedad en niños prematuros (6). El cuadro clínico consiste principalmente en meningitis, septicemia o enteritis necrozante en lactantes, aunque también se han observado cuadros diarreicos, infecciones urinarias y septicemia con niveles de letalidad asociadas a infección general de $26,9 \%$, con $42 \%$ y $20 \%$ en meningitis neonatal y septicemia respectivamente (7).

La enfermedad está asociada al consumo de leches en polvo rehidratadas (LP-R) como vehículo del patógeno con eventual participación de los utensilios y equipo como reservorios (8). Dado el carácter ubicuo del patógeno puede ser aislado de leche en polvo (LP) y rehidratada (LP-R), cereales infantiles, alimentos diversos, agua, superficies, hogares $y$ hospitales (9). Estudios sobre incidencia de Cronobacter spp en LP han demostrado su positividad en rangos de 3 a 30\% (10). En Chile, en un estudio realizado el año 2008 y publicado el año 2012 por Sáez y cols (11), encontraron 5\% de positividad de Cronobacter spp (Enterobacter sakazakii) en 80 muestras de LP de una planta elaboradora de productos lácteos de la Región de los Lagos.

La identificación actual de Cronobacter sakazakii se basa en la identificación mediante kits de test metabólicos (API 20E, ID32E, BIOLOG microarray) con bases de datos desactualizadas que reconocen aún a Enterobacter sakazakii. Además, mediante técnicas moleculares con sondas para reacción de la polimerasa en cadena (PCR) y tiempo real (PCR-RT) entre otras, que reconocen a Cronobacter spp y sus especies (12-15). Sin embargo, esta identificación se ve limitada al no considerar la reclasificación reciente de Cronobacter spp provocando falsos positivos (16). Baldwin y cols (17), mejoraron la identificación de Cronobacter spp y sus especies mediante multilocus sequence typing (MLST), que utiliza fragmentos de 7 house-keeping genes y que además, permite identificar en la especie C. sakazakii, ciertas predominancias genéticas asociadas al desarrollo de meningitis neonatal en niños (18). Para ello se utiliza la base de datos de acceso libre en: http:// www.pubMLST.org/cronobacter.

Considerando el riesgo asociado a Cronobacter, la FAO/ OMS han elaborado varias evaluaciones de riesgo con el fin de generar información para la toma de decisiones por parte de las autoridades de salud, además de recomendar la ausencia de Enterobacteriaceae, Cronobacter spp y sus especies en LP destinada al consumo de lactantes (19). Sin embargo, en Chile en las LP actualmente sólo se considera la ausencia de Salmonella en el Reglamento Sanitario de los Alimentos, no considerando Cronobacter spp y Enterobacteriaceae en su normativa.

El objetivo de este trabajo fue presentar resultados preliminares del proyecto DIUBB 143720 cuyo objetivo es evaluar el riesgo por C. sakazakii en LP destinadas al consumo de lactantes.

\section{MATERIALES Y MÉTODOS}

Muestreo: Se utilizó un estudio analítico de corte transversal desde agosto 2013 a Marzo de 2014. Se colectaron 72 latas (7 LP de prematuros y 65 de Etapa 1 o iniciación) de $450 \mathrm{~g}$ de 3 marcas y 3 países, comercializadas en farmacias y supermercados de Chillán. Como referencia se utilizaron los criterios de muestreo de la norma CAC/RPC 66 del Codex Alimentarius (20).

Microorganismos indicadores: Se utilizó el recuento de microorganismos mesófilos aerobios (RAM) y Enterobacteriaceae (ENT). La cuantificación de ambos grupos microbianos se realizó con la metodología descrita en el Compendium of Methods for the Microbiologycal Examination of Foods (21).

Aislamiento de Cronobacter spp: Se utilizó la técnica descrita por Iversen y Forsythe (22) utilizando 25 gramos de LP en $225 \mathrm{ml}$ de agua peptonada buffer (BPW, Oxoid, England). Se enriqueció en Caldo EE Mossel (BD Difco, Sparks, MD, USA) y posteriormente se estrió en Agar Cromogénico DFI (CM 1055, Oxoid Termo-Fisher, UK). Las colonias presuntivas (color verde o azul) se purificaron en agar soya tripticaseína (BD Difco, Sparks, MD, USA).

Identificación fenotípica: Se utilizó el kit comercial ID 32E (BioMérieux, Francia), siguiendo las indicaciones del fabricante.

Cuantificación por número más probable (NMP): Se utilizó la técnica descrita por Palcich y cols (23). Los límites se establecieron de 0,009 a $316 \mathrm{NMP} / \mathrm{g}$ de LP con un 95\% de confianza.

Amplificación para MLST: Se utilizó la metodología descrita en http://pubmlst.org/cronobacter. Todas las soluciones utilizadas de PCR CORE Kit Qiagen (Cat No. 201225). La secuenciación y purificación de los productos amplificados se realizó en MACROGENMR, Korea y Source Bioscience Sequencing, Nottingham, UK.

Identificación de Cronobacter sakazakii MLST: Este trabajo se realizó en el Pathogen Research Centre, Nottingham Trent University, Inglaterra. Identificando las cepas de Cronobacter sakazakii con los criterios de la base de datos de acceso libre http://pubmlst.org/cronobacter.

Análisis estadístico: Para describir se usaron medidas de tendencia central, dispersión y posición en el caso de variables cuantitativas, y frecuencias absolutas y porcentajes para variables cualitativas. Para comparar se utilizó la prueba de Mann- Whitney y Kruskall- Wallis utilizando el software STATA 7.0 con un nivel de significancia $\alpha=0,05$.

\section{RESULTADOS}

Para los recuentos de RAM, el 50\% de las muestras de LP etapa 1 tenían al menos $300 \mathrm{UFC/g}(10-36000)$ y de 650 UFC/g (70-30 000) para LP de prematuros. Por país de origen, se encontró un P50 global de 300 UFC/g (10-36 000), con un recuento para Chile $700 \mathrm{UFC/g}$ (30-36 000); México 220 UFC/g (10-5 000) y Holanda 500 UFC (70-30 000). Para la marca $n^{\circ} 1$ el P50 global muestra que la mitad de las muestras tiene al menos $700 \mathrm{UFC} / \mathrm{g}(30-36000), \mathrm{n}^{\circ}$ 2 de 170 UFC/g (10-30 000) y n 3 de 450 UFC/g (100-2 000). El recuento de RAM en las muestras procedentes de Chile, resultó ser significativamente mayor con respecto a los demás países en estudio $(p=0,016)$ al igual que la marca $n^{\circ} 1$ $(p=0,0069)$ (figura 1). El 92\% de las muestras evaluadas según este indicador y teniendo como referencia el RSA, cumplía con niveles microbiológicos aceptables (< $10000 \mathrm{UFC/g}$ ), $8 \%$ como medianamente aceptables ( $>10000 \mathrm{a}<50000$ UFC/g) y no existiendo muestras rechazables con valores > a $50000 \mathrm{UFC/g}$. Sólo cuatro muestras contenían probióticos con valores menores a $100 \mathrm{UFC/g}$.

Para los recuentos de ENT en LP etapa 1 el 50\% de las muestras contenían al menos 75 UFC/g (10-1 060) y 200 UFC/g (30-1 000) para LP de prematuros. Por país de origen, un P50 global fue de 90 UFC/g (10-1 060), siendo para Chile 160 UFC/g (10-1 060), México 60 UFC/g (10-1 000) y Holanda 150 UFC (10-1 000). Para la marca $n^{\circ} 1$, el P50 global muestra que la mitad de las muestras tenían $90 \mathrm{UFC/g}$ (10-1 060), la $\mathrm{n}^{\circ} 2$ con $60 \mathrm{UFC/g}(10-1000)$ y la $\mathrm{n}^{\circ} 3$ con $105 \mathrm{UFC} / \mathrm{g}(10-1$ 
000); no existiendo diferencias significativas en el recuento de ENT por tipo de leche $(p=0,097)$, país de origen $(p=0,086)$ y marca de LP $(p=0,158)$ (figura 2$)$. Considerando los rangos en los recuentos de ENT en LP de prematuros, 28,6\% (2/7) tenía menos de 100 UFC/g, 57,1\% (4/7) de 100 a 500 y $14,3 \%$ (1/7) con 1000 UFC/g. Para LP Etapa 1, 61\% de la LP contenía menos de $100 \mathrm{UFC} / \mathrm{g}, 36 \%$ entre 100 y 1000 y sólo 3\% con $1000 \mathrm{UFC} / \mathrm{g}$.

La prevalencia de C. sakazakii fue $2,7 \%$, siendo confirma- das sólo 2 cepas de lotes diferentes elaborados en Chile. Para las muestras producidas en Chile la prevalencia del patógeno fue $9,5 \%$ (tabla 1 ).

Se aislaron 20 cepas características en agar diferencial DFI de muestras manufacturadas en Chile, las que se identificaron como Enterobacter sakazakii mediante ID32E con 94 a 99.9\%. Además, se identificó en otras cepas a Enterobacter cloacae, Klebsiella pneumoniae y Enterobacter amnigenus. Para MLST sólo amplificaron 11 cepas, dos de las cuales fueron identifica-

\section{FIGURA 1}

Recuento de bacterias mesófilas aerobias (RAM) en leche en polvo por tipo de leche, país de origen y marca comercial.
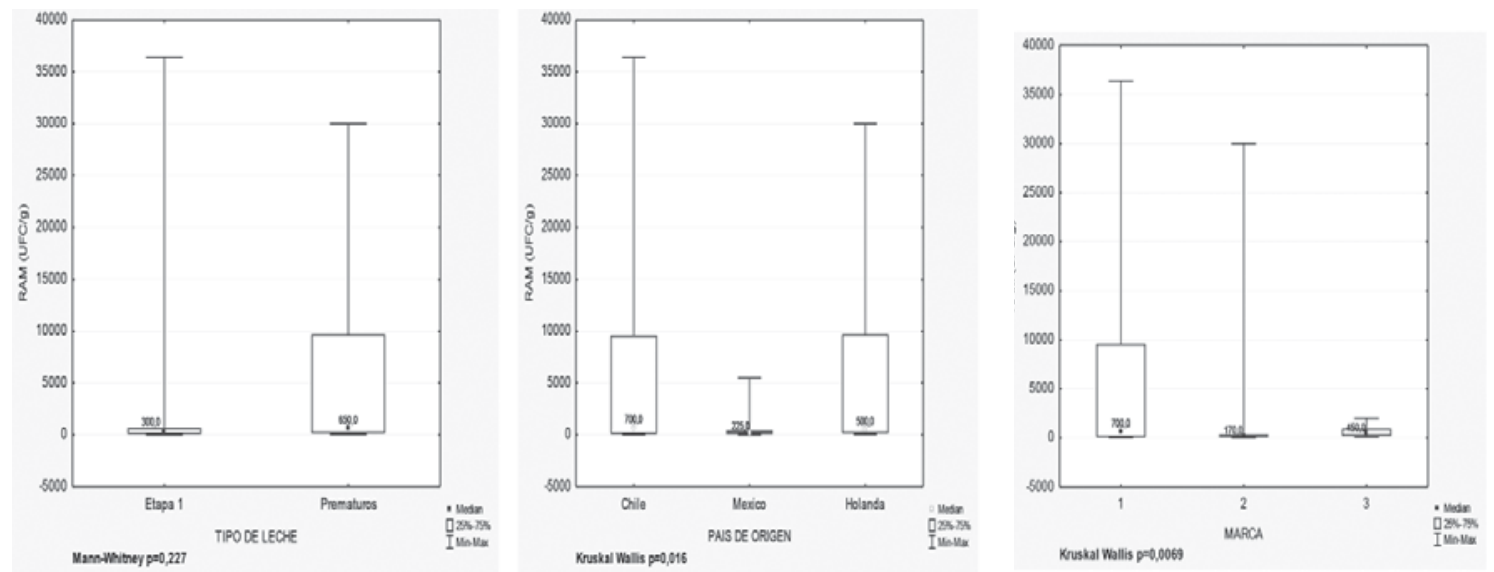

FIGURA 2

Recuento de Enterobacteriaceae en leche en polvo por tipo de leche, país de origen y marca comercial.
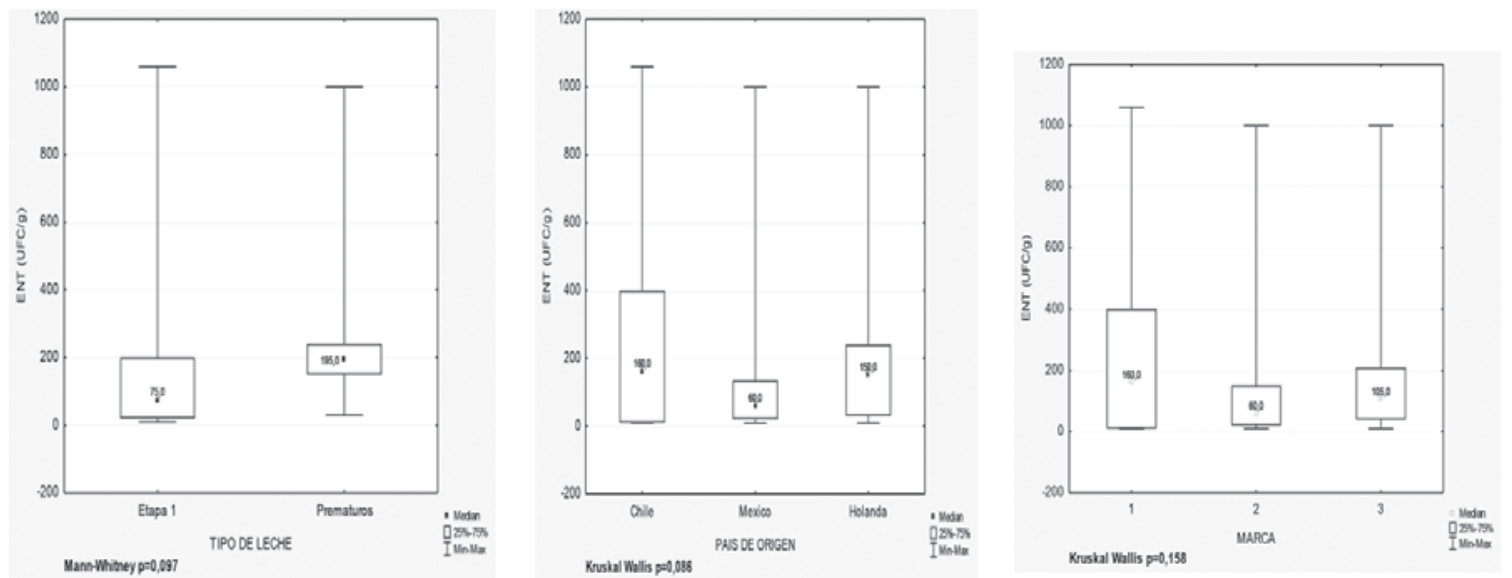
das como C. sakazakii con 0,23 y 2,3 NMP/g y Franconibacter helveticus con 0,023 y 0,94 NMP/g en otras dos. El resto de las cepas fueron identificadas como Enterobacter spp (tabla 2).

\section{DISCUSIÓN}

La lactancia materna es la forma más segura de alimentación para los lactantes: aporta la totalidad de los nutrientes necesarios para la primera etapa de la vida, protege contra las infecciones y tiene múltiples beneficios para la salud y desarrollo integral en los niños (24). La Organización Mundial de la Salud recomienda su consumo exclusivo hasta los 6 meses de vida y en combinación con otros alimentos, hasta los 2 años (25). Una alternativa a la lactancia materna son las leches en polvo (LP), que se obtienen a partir de leche de vaca y que por su proceso de elaboración industrial la población microbiana disminuye considerablemente. Por ello, estos productos no deben ser reconocidos como un producto estéril (26). De esta forma el uso de indicadores microbianos como recuento de aerobios mesófilos (RAM) y Enterobacteriaceae (ENT) nos proporciona información útil de las condiciones de higiene durante su elaboración.

De acuerdo al contenido de RAM en las muestras evaluadas, 92\% (66/72) de las muestras fueron calificadas como aceptables y $8 \%(6 / 72)$ medianamente aceptables según el
RSA. De las 6 muestras consideradas medianamente aceptables, cinco correspondieron a Chile. La otra muestra corresponde a LP elaborados en Holanda y destinada al consumo de niños prematuros. Estos valores de RAM son similares a los obtenidos por Chap y cols, que al estudiar 136 muestras de LP a nivel internacional encontraron que $75 \%$ de las muestras tenían menos de $100 \mathrm{UFC} / g, 16,2 \%$ entre 100 y 1000 UFC/g, $2.9 \%$ de 1000 a 10000 y 5,1\% con más de 100000 UFC/g. Evidenciando la necesidad de controlar las fuentes de contaminación de las LP debido al amplio rango de microorganismos presentes en el RAM y por la mayor susceptibilidad de infección que tienen los niños prematuros y lactantes (27).

Con respecto a ENT, $100 \%$ de las muestras analizadas presentaban valores sobre lo permitido según la norma internacional del Codex CAC RPC 66, que exige ausencia de este indicador en $25 \mathrm{~g}$ de LP. Estos resultados no se pueden comparar con el estándar de Chile ya que el RSA vigente no considera valores para este grupo indicador. Aún cuando, no existen diferencias significativas por tipo, marca o país de origen de las LP $(p>0,05)$, la sola presencia de Enterobacteriaceae en las LP es un factor de riesgo por sí solo. Esta situación no es del todo excepcional, Reich y cols en una planta de proceso de LP en Alemania, encontraron ENT en todas las muestras analizadas, y en siete lugares con valores muy superiores a 500

TABLA 1

Positividad de Cronobacter sakazakii en muestras de LP

\begin{tabular}{|c|c|c|c|c|c|}
\hline País & $n$ & $\begin{array}{c}\text { Prematuros } \\
(+)\end{array}$ & $\begin{array}{c}\text { Etapa } 1 \\
(+)\end{array}$ & $(+)$ & $\%$ \\
\hline Chile & 21 & NP & 2 & 2 & 9,5 \\
\hline México & 44 & NP & 0 & 0 & 0 \\
\hline Holanda & 7 & 0 & $\mathrm{NE}$ & 0 & 0 \\
\hline Total & 72 & 0 & 2 & 2 & 2,7 \\
\hline
\end{tabular}

TABLA 2

Identificación y cuantificación de C. sakazakii en leches en polvo mediante MLST.

\begin{tabular}{|c|c|c|c|c|}
\hline Cepa & $\begin{array}{l}\text { MLST } \\
\text { fusA }\end{array}$ & Identificación & $\begin{array}{l}\text { Cuantificación } \\
\text { (NMP/g) }\end{array}$ & Observaciones \\
\hline $\mathrm{CH} 3$ & 1 & C. sakazakii & 0,23 & \\
\hline $\mathrm{CH} 10$ & 71 & Franconibacter helveticus & 0,002 & Antes $C$. helveticus \\
\hline $\mathrm{CH} 18$ & 1 & C.sakazakii & 2,3 & \\
\hline $\mathrm{CH} 23$ & 58 & Enterobacters spp & 0,23 & \\
\hline $\mathrm{CH} 25$ & 58 & Enterobacter spp & & \\
\hline $\mathrm{CH} 26$ & 58 & Enterobacter spp & 0,23 & \\
\hline $\mathrm{CH} 30$ & 85 & Enterobacter spp & & \\
\hline $\mathrm{CH} 33$ & 85 & Enterobacter spp & & \\
\hline $\mathrm{CH} 34$ & 58 & Enterobacter spp & & \\
\hline $\mathrm{CH} 35$ & 71 & Franconibacter helveticus & 0,94 & Antes $C$. helveticus \\
\hline $\mathrm{CH} 41$ & 75 & Enterobacter spp & & \\
\hline
\end{tabular}


UFC/g. Enfatizando la necesidad del monitoreo en el proceso de elaboración y de medidas de inocuidad e higiene durante su producción (28).

La alta positividad a ENT en nuestro estudio es compatible con la presencia de varios microorganismos oportunistas y patógenos asociados a enfermedad en lactantes en diversas publicaciones (19). Por lo que estos hallazgos deben ser analizados en términos del riesgo asociado al consumo de las LP en lactantes, del poco control que estarían realizando las empresas elaboradoras y de la autoridad de salud responsable en nuestro país. Aun cuando, la asociación de enfermar con la ingesta de LP contaminados con ENT aún no se ha establecido con certeza, su ausencia en el LP proporciona una protección adicional a los recién nacidos, especialmente para prematuro, inmunocomprometidos, y recién nacidos de bajo (<2 $500 \mathrm{~g}$ ) y muy bajo (<1 $500 \mathrm{~g}$ ) peso al nacer, durante la preparación, almacenamiento, o la administración de la alimentación infantil (29).

Debido a la necesidad de asegurar la inocuidad de las LP en lactantes, la FAO/OMS ha realizado dos reuniones de expertos estudiando casos de enfermedades relacionadas a su consumo, ya sea epidemiológica o microbiológicamente. Se identificaron tres categorías de microorganismos con base en la solidez de las pruebas de una relación causal entre su presencia en los LP y la enfermedad de éstos: A) microorganismos con claras pruebas de causalidad, Salmonella entérica y Cronobacter (Enterobacter sakazakii); B) microorganismos para los cuales la causalidad es posible pero que no ha sido demostrada todavía, principalmente de la familia Enterobacteriaceae, y C) microorganismos en los cuales la causalidad es menos probable o no ha sido demostrada todavía, y que no han sido identificados en los LP (30). Las orientaciones entregadas por estas comisiones de expertos, a nuestro parecer fundamentan la necesidad de revisar los criterios microbiológicos del RSA vigente en términos de asegurar la inocuidad de las LP que se comercializan en nuestro país.

Además de encontrar otras especies de ENT en las LP, identificamos Cronobacter sakazakii en dos lotes de leches producidas en Chile. La presencia de este patógeno debe ser objeto de estudio y fiscalización por parte del fabricante y de las autoridades, debido a su alta letalidad y secuelas neurológicas que produce (31). La probabilidad de enfermar por este patógeno ha sido estimada en muy pocos países. En Estados Unidos se estima una tasa de infección de 1 por 100 000 niños recién nacidos y que aumenta a 9,4 por 100000 en niños con un peso menor a 1,500 g (32). En Holanda, se estima una probabilidad de 0,53 casos de infección por año con una tasa de 1 por 100000 niños (33). En Chile desconocemos esta información, ya que $C$. sakazakii no es parte de la pesquisa habitual de notificación.

A fines de 2011 en Estados Unidos, 4 niños menores de 6 meses enfermaron gravemente de meningitis al consumir LP contaminada por C. sakazakii de los cuales dos murieron. Se identificó la presencia en las cepas aisladas del complejo clonal ST4 que ha sido asociado al desarrollo de meningitis neonatal y que está presente en todos los casos de meningitis asociada a este patógeno en los últimos 30 años (34).

La cuantificación de este patógeno en una de las muestras fue $2.3 \mathrm{NMP} / \mathrm{g}$, valor muy elevado de acuerdo a lo que reporta la literatura científica (19). Si consideramos que un lactante consume varias veces al día y diferentes volúmenes, el riesgo aumenta. Aun cuando la dosis infectante es desconocida, Iversen y cols, propusieron 1000 UFC (35), diferente de expresada por la OMS de 10000 UFC (19).
Además, identificamos en 2 cepas de dos muestras producidas en Chile a Franconibacter helveticus, germen conocido hasta julio 2014 como Cronobacter helveticus, especie estrechamente relacionada a Cronobacter spp (36),

Otro aspecto importante a considerar es que desde el año 2007, la OMS recomienda el uso de agua $>70^{\circ} \mathrm{C}$ para hidratación de las leches en polvo para limitar el riesgo de infección por Cronobacter spp (37). Recomendando además, que la administración de los LP rehidratados a los lactantes se realice dentro de 2 horas después de preparado o conservado en refrigeración $\mathrm{a}<\mathrm{de} 4^{\circ} \mathrm{C}$. No cumplir con esta temperatura de refrigeración le confiere la posibilidad de desarrollo al patógeno por su característica psicrótrofa (38).

Ninguna de las etiquetas de las LP evaluadas en nuestro estudio consideraba este aspecto, y solo recomendaba el uso de agua hervida tibia, lo que no sólo contraviene la recomendación de OMS, sino que expone a los lactantes a un riesgo innecesario. Forsythe (2009), encontró en un estudio extensivo en Europa que las etiquetas de las LP no entregan instrucciones de reconstitución con agua a $70^{\circ} \mathrm{C}$, sólo especifican el uso de agua de rehidratación hervida y tibia, siendo riesgo permanente de desarrollo de Cronobacter y otras enterobacterias (39).

En resumen, en este estudio encontramos una inadecuada calidad microbiológica de las LP que son consumidas por prematuros y lactantes. Además, identificamos la presencia de Enterobacteriaceae y de Cronobacter sakazakii, lo que es un llamado de alerta a los fabricantes y autoridades reguladoras en la salud pública de nuestro país. Es necesario establecer un mejor control de las condiciones higiénicas en la elaboración de las LP y de su vigilancia microbiológica, con el fin de evitar riesgos innecesarios para la salud en la población infantil que consume masivamente estos alimentos.

\section{RESUMEN}

Introducción: Cronobacter spp es un género bacteriano con 7 especies, siendo C. sakazakii la especie clínica más reportada y asociada a meningitis y septicemia en lactantes. Es transmitida por leche en polvo (LP) por lo que la OMS recomienda su ausencia en este producto. En Chile, el reglamento sanitario de los alimentos (RSA) no lo considera. Objetivo: Evaluar el riesgo por Cronobacter sakazakii en LP destinadas al consumo de lactantes. Metodología: Se analizaron 72 muestras de LP de 3 marcas y 3 países. El recuento de bacterias mesófilas (RAM), Enterobacteriaceae (ENT) y número más probable (NMP) se realizó con la metodología de Puch and Ito (2001). Se utilizó agar diferencial Cronobacter para aislamiento (DFI, Oxoid, England) y kit bioquímico ID32E (Biomeriux, Francia) para fenotipo. El patógeno fue identificado y genotipificado por multilocus sequence typing (MLST) utilizando criterios de http://www.pubmlst.org/cronobacter. Resultados: La medianas de RAM para LP etapa 1 y prematuros fueron $300 \mathrm{UFC/g}$ (10-36 000) y $650 \mathrm{UFC/g}(70-30000)$, siendo mayor en las muestras de Chile $(\mathrm{p}=0,016)$. Para ENT de $75 \mathrm{UFC} / \mathrm{g}(10-1060)$ y 200 UFC/g (30-1 000), no existiendo diferencias significativas por tipo, país o marca de LP $(p>0,05)$. Dos cepas de 2 lotes diferentes características en agar DFI se identificaron como $C$. sakazaki con 0,23 y 2,3 NMP/g. Además de Franconibacter helveticus en otras 2 cepas, especie relacionada estrechamente con Cronobacter spp. Conclusiones: La prevalencia de C. sakazakii en todas las muestras fue de $2,7 \%$ y aisló sólo en LP de elaborados en Chile. La ausencia de Cronobacter spp en $25 \mathrm{~g}$ debe ser incorporado en el RSA de Chile.

Palabras clave: Cronobacter sakazakii, leche en polvo, lactantes. 
Agradecimientos: A la Universidad del Bío-Bío, Chile (proyecto DIUBB 143720), Santander Research Mobility Grant y Pathogen Research Centre, Nottingham Trent University, Inglaterra, por su apoyo en la ejecución de este proyecto.

\section{BIBLIOGRAFÍA}

1. Koletzko B, Shanir R, Ashwell M, and early Nutrition academy. Quality and Safety Aspects of Infant Nutrition. Ann Nutr Metab. 2012; 60:179-84.

2. Townsend S, Hurrell E, and Forsythe S. Virulence studies of Enterobacter sakazakii isolates associated with a neonatal intensive care unit outbreak. BMC Microbiol. 2008; 8:64.

3. Farmer JJ, Asbury M, Hickman F, Brenner D. The Enterobacteriaceae Study Group. 1980. Enterobacter sakazakii, new species of Enterobacteriaceae isolated from clinical specimens. Int J Syst Bacteriol. 1980; 30:569-84.

4. Iversen C, Mullane N, Mc Cardell B, Tall B, Lehner A, Fanning $S$, Stephan $R$, Joosten $H$. Cronobacter gen. nov., a new genus to accommodate the biogroups of Enterobacter sakazakii, and proposal of Cronobacter sakazakii gen. nov. comb. nov., C. malonaticus sp. nov., C. turicensis sp. nov., C. muytjensii sp. nov., C. dublinensis sp. nov., Cronobacter genomospecies 1 , and of three subspecies, C. dublinensis sp. nov. subsp. dublinensis subsp. nov., C. dublinensis sp. nov. subsp. lausannensis subsp. nov., and $C$. dublinensis sp. nov. subsp. lactaridi subsp. nov. Int I Syst Evol Microbiol. 2008; 58:1442-7.

5. Joseph S, Cetinkaya E, Drahovska H, Levican A, Figueras $M$ and Forsythe S. Cronobacter condimenti sp. Nov., isolated from spiced meat, and Cronobacter universalis sp. Nov., a species designation for Cronobacter sp. Geneomoespecies 1, recovered from a leg infection, water and food ingredients. Int J Syst Evol Microbiol. 2012; 62:1277-83.

6. Jason J. Prevention of invasive Cronobacter infection in young infants fed powdered infant formulas. Pediatrics. 2012; 130:1076-84.

7. Holý $O$, Forsythe S. Cronobacter spp as emergent causes of healthcare-associated infection. J Hosp Infect. 2014; 86(3): 169-7.

8. Jackson E, Sonbol H, Masood N, Forsythe S. Genotypic and phenotypic characteristics of Cronobacter species, with particular attention to the newly reclassified species $C$. helveticus, C. pulveris, and C. zurichensis. Food Microbiol. 2014; 44:226-35.

9. Baumgartner $A$, Grand $M$, Liniger $M$ and Iversen C. Detection and frequency of Cronobacter spp (Enterobacter sakazakii) in different categories of ready-to-eat foods other than infant formula. Int J Food Microbiol. 2009; 136:154-9.

10. Corli Witthuhn $R$, Kemp F, Britz TJ. Isolation and PCR detection of Enterobacter sakazakii in South African food products, specifically infant formula milks. World J Microbiol Biotechnol. 2007; 23:151-7.

11. Saéz M, Llanos S, Tamayo R. Primer aislamiento de Cronobacter spp (Enterobacter sakazakii) en fórmula láctea en polvo producida en Chile. Rev Ch Salud Púb. 2012; 16(1):11-5.

12. Lehner A, Tasara T and, Stephan R. 16S rRNA gene based analysis of Enterobacter sakazakii strains from different sources and development of a PCR assay for identification. BMC Microbiol. 2004; 4:43-9.

13. Wang, $X, Z$ Zhu $C, X u X$, Zhou G. Real-time $P C R$ internal amplification control for the detection of Cronobacter spp
(Enterobacter sakazakii) in food samples. Food Control. 2012; 25:144-9.

14. Hassan A, Akineden O, Kress C, Estuningsih S, Schneider $E$ and, Usleber E. Characterization of the gene encoding the 16S rRNA of Enterobacter sakazakii and development of a species specific PCR method. Int I Food Microbiol. 2007; 116:214-20.

15. Stoop B, Lenher $A$, Iversen C, Fanning S. Development and evaluation of $r p o B$ based $P C R$ systems to differentiate the six proposed species within the genus Cronobacter. Int J Food Microbiol. 2009; 136:165-8.

16. Cetinkaya E, Joseph S, Ayhan K and Forsythe S. Comparison of methods for the microbiological identification and profiling of Cronobacter species from ingredients used in the preparation of infant formula. Mol Cell Probes. 2012; 27:60-4.

17. Baldwin A, Loughlin M, Caubilla-Barron J, Kucerova E, Manning $G$, Dowson $C$ and Forsythe S. Multilocus sequence typing of Cronobacter sakazakii and Cronobacter malonaticus reveals stable clonal structures with clinical significance, which do not correlate with biotypes. BMC Microbiol. 2009; 9:223.

18. Joseph S and Forsythe S. 2011. Predominance of Cronobacter sakazakii sequence type 4 in neonatal infections. Emerg Infect Dis. 2011; 17(9):1713-5.

19. FAO/WHO. Enterobacter sakazakii (Cronobacter spp) in powdered follow-up formulae. Microbiological Risk Assessment Series No.15. Rome. 2008.

20. CODEX ALIMENTARIUS. Código de prácticas de higiene para los preparados en polvo para lactantes y niños pequeños. CAC/RCP 66. 2008

21. Compendium of methods for the Microbiological Examination of Foods. APHA. Pouch Downes and Ito ed. USA. 2001.

22. Iversen C, Forsythe S. Isolation of Enterobacter sakazakii and other Enterobacteriaceae from powdered infant formula milk and related products. Food Microbiol. 2004; 21: 771-6.

23. Palcich G, Moraes L, Casale L, Pagotto F, Farber J, Landgraf $M$, and Destro MT. Enterobacter sakazakii in dried infant formula and milk kitchens of maternity wards in Sao Paulo, Brasil. J Food Prot. 2009; 72(1): 37-42.

24. Vargas-Leguás $H$, Rodríguez $V$, Lorite $R$, Pérez-Portabella C, Redecillas S, Campins M. Guía para la elaboración de fórmulas infantiles en polvo en el medio hospitalario. Sistema de análisis de peligros y puntos de control crítico. An Pediatr. 2009; 70 (6):586-93.

25. WHO (Organización Mundial de la Salud): 54th World Health Assembly, Infant and young child nutrition. WHA 54.2, May 18, 2001. http://apps. who.int/gb/archive/pdf_ files/WHA54/ea54r2.pdf (accesada 23 noviembre 2014).

26. Gurtler J, Beuchat L. 2007. Growth of Enterobacter sakazakii in reconstituited infant formula as affected by composition and temperature. J Food Prot. 2007; 70:2095-210.

27. Chap J, Jackson P, Siqueira R, Gaspar N, Quintas C, ParkJ, Osaili T, Shaker R, Jaradat Z, Hartantyo S, Abdullah Sani $N$, Estuningsih $S$ and Forsythe $S$. International survey of Cronobacter sakazakii and other Cronobacter spp. in follow up formulas and infant foods. Int I Food Microbiol. 2009; 136:185-8.

28. Reich $F$, König $R$, von Wiese $W$, Klein G. Prevalence of Cronobacter spp. in a powdered infant formula processing environment. Int J Food Microbiol. 2010; 140:214-7.

29. Abdullah Sani N, Hartantyo S, Forsythe S. Microbiological assessment and evaluation of rehydration instructions on 
powdered infant formulas, follow-up formulas and infant foods in Malaysia. J Dairy Sci. 2013; 96:1-8.

30. Siqueira RF, da Silva $N$, Junqueira V, Kajsik M, Forsythe $S$, Pereira J. Screening for Cronobacter species in powdered and reconstituted infant formulas and from equipment used in formula preparation in maternity hospitals. Ann Nut Met. 2013; 63:62-8.

31. Lai $K$. Enterobacter sakazakii infections among neonates, infants, children, and adults. Case reports and review of the literature. Medicine. 2001; 80:113-2.

32. Kucerova E, Joseph S, Forsythe S. The Cronobacter genus: ubiquity and diversity. Qual Assur Safety Foods Crops. 2011; 3:104-22.

33. Reij $M$, Jongerburger I, Gkogka E, Gorris L, Zwietering $M$. Perspective on the risk to infants in the Netherlands associated with Cronobacter spp. occurring in powdered infant formula. Int J Food Microbiol. 2009; 136:232-7.

34. Hariri S, Joseph S, Forsythe S. Cronobacter sakazakii ST4 strains and neonatal meningitis, United States. Emerg Infect Dis. 2013; 19: 175-7.

35. Iversen $C$, Lane $M$, Forsythe $S$. The growth profile, thermotolerance and biofilm formation of Enterobacter sakazakii grown in infant formula milk. Lett Appl Microbiol. 2004;

\section{8:378-82.}

36. Stephan R, Grim C, Gopinath G, Mammel M, Sathyamoorthy $V$, Trach L, Chase H, Fanning S, Tall B. Re-examination of the taxonomic status of Enterobacter helveticus, Enterobacter pulveris and Enterobacter turicensis as members of the genus Cronobacter and their reclassification in the genera Franconibacter gen. nov. and Siccibacter gen. nov. as Franconibacter helveticus comb. nov., Franconibacter pulveris comb. nov. and Siccibacter turicensis comb. nov., respectively. Int J Syst Evol Microbiol. 2014;10: 3402-10.

37. World Health Organization. 2007. Safe preparation, storage and handling of powdered infant formula. Available at: http://www.who.int/foodsafety. Accessed 05 November 2014.

38. Flores JP, Arvizu S, Silva J, Fernández E. Two cases of hemorrhagic diarrhea caused by Cronobacter sakazakii in hospitalized nursing infants associated with the consumption of powdered infant formula. J Food Prot. 2011; 74(12): 2177-81.

39. Forsythe S. J. 2009. Bacteriocidal preparation of powdered infant formula. Avalaible at: http://www.foodbase.org. uk/results.php?f_report_id=395. Accessed 23 Noviembre 2014. 